FACTA UNIVERSITATIS

Series: Mechanical Engineering Vol. 19, Nº 1, 2021, pp. 23 - 38

https://doi.org/10.22190/FUME210108021C

Original scientific paper

\title{
A FRACTURE-INDUCED ADHESIVE WEAR CRITERION AND ITS APPLICATION TO THE SIMULATION OF WEAR PROCESS OF THE POINT CONTACTS UNDER MIXED LUBRICATION CONDITION
}

\author{
Hui Cao, Yu Tian, Yonggang Meng
}

State Key Laboratory of Tribology, Tsinghua University, China

\begin{abstract}
Adhesive wear is one of the four major wear mechanisms and very common in almost all macro-, micro- or nanotribosystems. In an adhesive wear process, tiny material fragments are pulled off from one sliding surface and adhered onto the counterpart. Later these fragments form loose particles or transfer between the contact surfaces. Because of the topographical and physicochemical property non-uniformity of engineering surfaces, adhesive wear happens heterogeneously on the loaded sliding surfaces, and it is also discontinuous during sliding or rolling motion owing to the damage accumulation and fracture occurred inside the subsurface layers. Taking account of these characteristics, a novel fracture-induced adhesive wear criterion has been proposed in this study in order to predict local wear of material in sliding. Moreover, the proposed wear criterion is applied to predicting wear particle formation and morphology evolution of mixed lubricated rough surfaces during reciprocating sliding, and the simulation results are compared with the ball-on-disk experimental measurements.
\end{abstract}

Key Words: Adhesive wear, Criterion, Mixed lubrication, Surface energy, Wear particles

\section{INTRODUCTION}

Wear of material is ubiquitous in almost all macro-, micro- or nano systems in motion. Usually, it has significant unfavorable influences on operating performances and lives of devices and machinery, such as smearing or fatigue failures frequently occurring in high speed rolling bearings [1]. In some cases, wear could bring about benefits, which are not attainable by other ways, to tribosystems. For instance, an appropriate running-in wear process could lead to a super low coefficient of friction [2] or a stable low wear rate [3]. Ultra-flat and ultra-smooth solid surfaces, which are required in manufacturing of modern integrated microelectronic and

Received January 08, 2021 / Accepted February 19, 2021

Corresponding author: Yonggang, Meng

Affiliation: State Key Laboratory of Tribology, Tsinghua University, Beijing, 100084, China

E-mail: mengyg@tsinghua.edu.cn 
photonic devices, are made with the technology of chemical mechanical polishing process that needs delicate balance between corrosion and mechanical wear [4].

Wear is a multiscale and multiphysics phenomenon. There are many intrinsic and extrinsic factors affecting wear behavior of materials. Although scientific study on wear can be dated back to Holm in 1946 [5], and a tremendous experimental and theoretical effort has been paid since then to the wear problems, it is still impossible to predict formation and evolution of wear debris particles as well as wear life of machine elements in an engineeringly acceptable accuracy. In 1995, Meng and Ludema [6] reviewed the published work on wear models and predictive equations, and they disappointedly concluded that none of the over 300 equations proposed could ever be found for general and practical use. Most of the wear equations are empirical regression relationships between total macroscopic material loss (in terms of mass, volume or depth) and operation parameters including load and speed, such as that proposed by Rhee [7], based on experimental data collected in a limited range of test conditions for a specific application, regardless of wear mechanism involved and microscopic debris formation. There are also some mechanism-based wear models; the most famous and widely accepted one of them is the Archard law which states that wear volume is proportional to normal load and sliding distance and so is inverse proportion to the hardness of the softer material of contacting bodies [8]. The Archard law is derived from the assumptions of adhesive wear occurring at microscopic asperity contacts of rough surfaces and hemispherical shape of wear particles, but it tells nothing about size and number of wear particles. Later, a number of variants of Archard law are proposed, each presenting a different expression of wear coefficient. In 1961, Rabinowicz presented a surface energy criterion for loose of adherent wear particles from solid surfaces [9]. He postulated that loose of adherent wear fragments from surfaces was caused by the release of elastic energy stored in the compressed fragments from a contacted state to a non-contacted state. Under the assumption of hemispherical shape of wear fragments, he derived a critical size, $\mathrm{d} L$, which is proportional to the ratio of work of adhesion to hardness. Adherent fragments smaller than the critical size could not get loose from surfaces. He indicated that the critical size could predict equilibrium surface roughness after sliding test, asperity junction size, the minimum load effect on wear as well as the minimum size of wear particles [10]. N. P. Suh et al. developed a delamination wear model based on contact fatigue mechanism [11]. Quinn et al. proposed a mechanism of surface oxidation wear and believed that excessive surface temperature rise in friction process would lead to the oxidation of surface materials. When the oxidation layer reaches a critical thickness, it would automatically loose off from the substrate [12].

In the last two decades, along with the progress in experimental techniques with micro-nanoscale resolutions [13-16], atomic simulations [17-19], discrete dislocation dynamics of crystal materials [20][21] as well as contact, damage and fracture mechanics, understanding of microscopic wear mechanisms and modeling of wear process have developed greatly. Distinctions between atomic adhesive wear, plastic wear and fracture-induced adhesive wear have been identified, and the size effect on friction and wear is emphasized more and more. Recent coarse grain molecular dynamics (CGMD) simulations [18] have shown that transition from plastic deformation to fractured-induced wear occurs when the junction size exceeds a critical length scale, a re-finding of the critical size concept proposed by Rabinowicz based on his surface energy criterion. Popov and Pohrt [22] extended the Rabinowicz criterion to asperity-free case and numerically modeled wear particle emission of dry contacts with the boundary element method. Tan et al. proposed that asperity wear is caused by fatigue due to multiple collisions during friction, and deduced formulas for calculating the macroscopic wear 
of a rough surface combined with statistical contact model [23]. However, these wear modeling and simulations neglected the effects of lubrication.

Undoubtedly, lubrication affects adhesive wear greatly. Firstly, a little bit of adsorbed molecular on surfaces, even a small quantity of contaminants, could reduce adhesion strength of contacting surfaces significantly. Secondly, most of formulated oils used in industry contain some amount of friction modifiers and antiwear additives which could form boundary lubrication films and reduce wear. Thirdly, fluid film lubrication can reduce frictional heating and thus suppress temperature rise of surfaces during sliding, leading to weaker adhesion compared with dry friction. Last but not least, hydrodynamic fluid film and boundary film can support a part of or even the whole of the applied load, substantially reducing or even eliminating asperity junctions. To account the effects of fluid film and boundary film on wear, wear modeling should be coupled with the theory and numerical analysis of lubrication properly. On one hand, lubrication alters wear resistance of tribopairs, while, on the other hand, wear brings about nonrecoverable changes in the shape and topography of sliding bodies, which affects lubrication film formation and hydrodynamic pressure. Although the theory and numerical modeling of hydrodynamic and elastohydrodynamic lubrication have well developed since the foundation of Reynolds equation in 1886, the basic assumption of no wear is still adopted in most of the lubrication simulations, even for the cases of mixed lubrication.

In this study, a novel criterion for fracture-induced adhesive wear is proposed, taking into account the effects of work of adhesion and surface energy degradation on adhesive wear. Moreover, the stochastic distributions of these physical properties of materials are also considered in the wear criterion. The proposed wear criterion is applied to predict the wear process of a mixed lubricated point contact in sliding motion. Morphology evolution of surfaces and wear particles formation during wear process are simulated and compared with experiment measurements.

\section{A FrACTURE-INDUCED ADHESIVE WEAR CRITERION}

\subsection{Assumptions of fracture-induced adhesive wear process}

As mentioned above, fracture-induced adhesive wear dominates when the junction size exceeds a critical length scale. From the perspective of material damage mechanism, fracture-induced wear is a process during which the surface and subsurface are gradually damaged and accumulated to a certain extent under repeated external load and frictional force, resulting in the shedding of tiny material from the substrate. Generally, it can be divided into brittle damage and ductile damage. However, no matter what type of damage is, chemical bonds between atoms or molecule break, or de-cohesion occurs, and new surfaces are generated if wear of materials happens. Therefore, the essence of the fracture-induced wear process can be considered as accumulation of the damages in the surface layer and subsurface layer, and finally a piece of material falls off from the matrix to form new surfaces owing to the input of a part of external mechanical energy into the frictional system. The other parts of external mechanical energy dissipate as thermal energy and other forms of energy. The surface energy degradation here is a comprehensive description of the decrease in the external work needed to generate new surfaces. Its essence is that the input frictional work continually leads to nucleation and propagation of subsurface cracks, and hence the energy needed to separate local materials from the substrate decreases. For example, the micro-cracks initially generated in the subsurface layer 
propagate gradually under continuous frictional work, and the slip lines in the subsurface layer gradually tighten and intersect [24], which results in the micro-wear particles naturally falling off from the substrate or are stuck away by the aid of work of adhesion. Therefore, wear process is also affected by the adhesive behaviors of contact surfaces, especially for metals. Therefore, work of adhesion plays a vital role in adhesive wear [25].

\subsection{A new fracture-induced adhesive wear criterion}

It is often observed in frictional processes that some asperities are worn off in a relatively short rubbing time while others require a relative longer period. Besides the differences in local pressure acting at different asperities, it can be attributed to variations of adhesion strength and material properties over the sliding surface. Hence, local wear tends to be discontinuous in space and time. Moreover, wear thickness also presents strong randomness. Therefore, it can be reasonably speculated that the wear of material happens in the form of removal of fragments with random thickness far greater than the single atomic layer at different time intervals under the action of friction. The shapes and sizes of wear particles display statistical distributions as shown in Fig. 1.

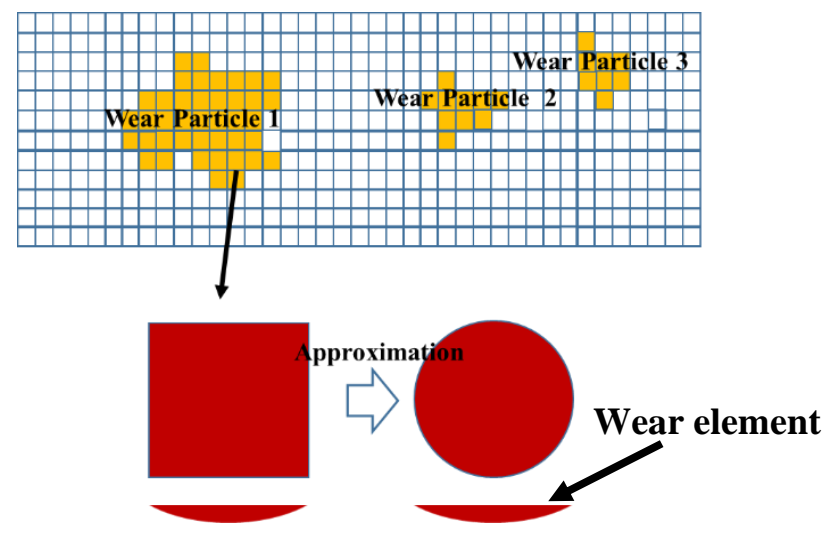

Fig. 1 Schematic of wear particles and wear element

It is worth noting the distinction between a wear particle and a wear element, a concept introduced in our model, as shown in Fig. 1. Wear element is defined as the basic minimum unit of wear particles. A cluster of inter-connecting wear elements constitutes a wear particle. The size of a wear element is related to the discretization mesh which should be small enough to depict surface roughness but larger than the critical length scale beyond which fracture-induced wear dominates as mentioned above. The shape of a wear element is assumed as a convex spherical crown with a projection diameter of the mesh size for simplicity. Therefore, in simulations based on the wear criterion, when a wear element is formed and removed at a grid point, the surface height changes by a decrement that equals the height of the convex spherical crown at that grid point. Unlike the Archard law or many previously proposed wear models where wear particle size is assumed to be equal to the junction diameter, here the size of wear elements rather than wear particles is presumed. At a certain moment $\mathrm{t}$ during sliding, whether the element located at $(x, y)$ on the sliding surface is worn off or not is judged by the following criterion. 


$$
\theta=\frac{A_{c} W_{12}(x, y, t)}{2\left[\gamma(x, y)-\int_{0}^{t} \alpha \mu_{c} p_{\mathrm{c}}(x, y, t)\left|u_{2}-u_{1}\right| \mathrm{d} t\right] A_{\mathrm{w}}}
$$

If $\theta \geq 1$, the element is worn off, otherwise no wear element forms but the material degradation progresses. The criterion in Eq. (1) expresses the competition between work of adhesion at the interface and the increase in surface energy accompanied with the generation of a wear element. Wear occurs at the location $(x, y)$ only when the work of adhesion exceeds or equals the increase in surface energy of the system as a result of falling off the element from the substrate. Therefore, the criterion gives the necessary condition for a wear element to generate. However, the criterion is not concerned with any behavior of a wear element after it is generated. In other words, it is assumed that all generated wear elements disappear from the tribosystem after their birth. Therefore, the effect of wear particles in the contact region could not be evaluated by using this criterion. In following, the parameters in criterion (1) will be described in detail.

The numerator is the product of projection area, Ac, and the work of adhesion per unit area $W_{12}(x, y, t)$ at location $(x, y)$ in time t. If there is no adsorption or reaction films at the interface, the work of adhesion, $W_{12}$, is the sum of the surface energy per unit area of the two surfaces in contact minus the interfacial energy of the two surfaces, as shown in Eq. (2), where the surface energy is a material property, and the interfacial energy depends on the compatibility between the contacting bodies. Factor $\mathrm{cm}$ denotes the magnitude of compatibility and takes a value in the range from 0 to 0.5 [26]. However, for lubricated contacts, work of adhesion changes substantially depending on the local lubrication state. In mixed lubrication, direction contacts without any intervening layer, contacts with a boundary film and contacts separated by a fluid film may co-exist on the sliding surface, and thus work of adhesion probably takes a random value in the range $\left[W_{12 \min }, W_{12 \max }\right]$ as expressed in Equation (3), where $U$ means uniform distribution.

$$
\begin{gathered}
W_{12}(x, y)=\gamma_{1}(x, y)+\gamma_{2}(x, y)-\gamma_{12}(x, y)=c_{m}\left(\gamma_{1}(x, y)+\gamma_{2}(x, y)\right) \\
W_{12}(x, y) \sim U\left(W_{12 \min }, W_{12 \max }\right)
\end{gathered}
$$

The bracket in the denominator of Equation (1) represents the monotonic degradation of surface energy from its initial value of $\gamma(x, y)$, owing to the accumulation of input frictional work done during the time period $[0, t]$. When a wear element forms, a convex spherical crown surface and a concave spherical crown surface with the same area of $A_{\mathrm{w}}$ are generated at the same time, so the new surface area in the denominator of Eq. (1) is 2 times the spherical crown area. For most engineering materials, the surface energy on sliding surface is in general nonhomogeneous. Here we assume that the initial surface energy per unit area obeys the normal random distribution as expressed in Eq. (4), where $N\left(\gamma_{\mathrm{m}}, \sigma_{\gamma}\right)$ means the normal distribution with the mean value of $\gamma_{\mathrm{m}}$ and standard deviation $\sigma_{\gamma}$.

$$
\gamma(x, y) \sim N\left(\gamma_{\mathrm{m}}, \sigma_{\gamma}\right)
$$

The integration in the bracket represents the accumulative degradation of surface energy at $(x, y)$ under contact pressure $p_{\mathrm{c}}$, relative sliding speed $\left|u_{1}-u_{2}\right|$ and frictional coefficient $\mu_{\mathrm{c}}$ during the time period $[0, t]$. Factor $\alpha$, referred as conversion coefficient, means how much of the frictional work dissipated as the surface energy degradation. It is a characteristic parameter of 
individual material, and can be estimated experimentally or by atomic simulations. Methods for determination of $\alpha$ and calculation of $p_{\mathrm{c}}$ will be described in the next section.

Another key parameter that does not explicitly appear in the criterion (1) but is of importance for prediction of wear volume and morphology evolution is the thickness of wear element. For fracture-induced adhesive wear, the thickness of wear element corresponds to the position within the subsurface layer where crack nucleation and propagation are most probable. Considering various intrinsic and extrinsic influence factors, including the microstructures, inclusions and defects distributions in materials produced in manufacturing processes as well as working conditions during service, in this study, the thickness of wear element is assumed to yield a continuous lognormal distribution with a mean value of $\Delta_{\mathrm{m}}$ and a standard deviation of $\sigma_{\Delta}$, as following.

$$
\ln \Delta \sim N\left(\ln \Delta_{\mathrm{m}}, \ln \sigma_{\Delta}\right)
$$

In the next sections, the proposed wear criterion is applied to a reciprocating ball-on-plate experiment, and the predicted evolution of surface topography of the plate in wear process is compared with measurements.

\section{Simulation OF WEAR EVOLUTION UNDER MiXED LUBRICATION CONDITION AND COMPARISON WITH EXPERIMENT}

\subsection{Simulation procedure}

Fig. 2 shows the flow chart used to simulate the wear process of a ball-on-plate sliding friction test in mixed lubrication condition, the details of which are to be described below in Section 3.4. The upper sample was a bearing steel ball with a diameter of $12.7 \mathrm{~mm}$, and the lower sample was a carbon steel plate. The original surface of the steel plate was a ground surface with root mean square roughness $\mathrm{Rq}$ of $0.21 \mu \mathrm{m}$, while the initial surface roughness $R \mathrm{q}$ of the steel ball is $0.014 \mu \mathrm{m}$, more than 10 times smaller than that of the plate. Since the ball was much harder and smoother than the plate, the roughness effect of the ball on lubrication and wear was neglected, and the wear of the ball was not accounted in the simulation. Reciprocating friction simulation was carried out across a stroke (about double of Hertz contact zone) compatible with the optical field size of the white light interferometer used, for comparison with measurement to be described later.

The wear simulation process is a step-by-step repeat of mixed lubrication numerical analysis, updating the 3D surface topography and material properties such as surface energy at every grid point $(x, y)$ according to the situations whether the wear element at that point breaks away the plate surface or not, judged by the wear criterion (1) after an increment of time step for wear updating. The unified Reynolds equation established by $\mathrm{Hu}$ and Zhu [26] was used to deal with the mixed lubrication problems of point contacts. To explore the wear particle emission and surface topography evolution in detail, $512 \times 512$ finite difference grids, corresponding a mesh size of $1.17 \mu \mathrm{m}$, were used in numerical simulation, which is denser than conventional mixed lubrication modeling without accounting wear particle generation. Considering balance of computational efficiency with accuracy, time step $\Delta t$ for wear updating was set as 50 reciprocating cycles. 


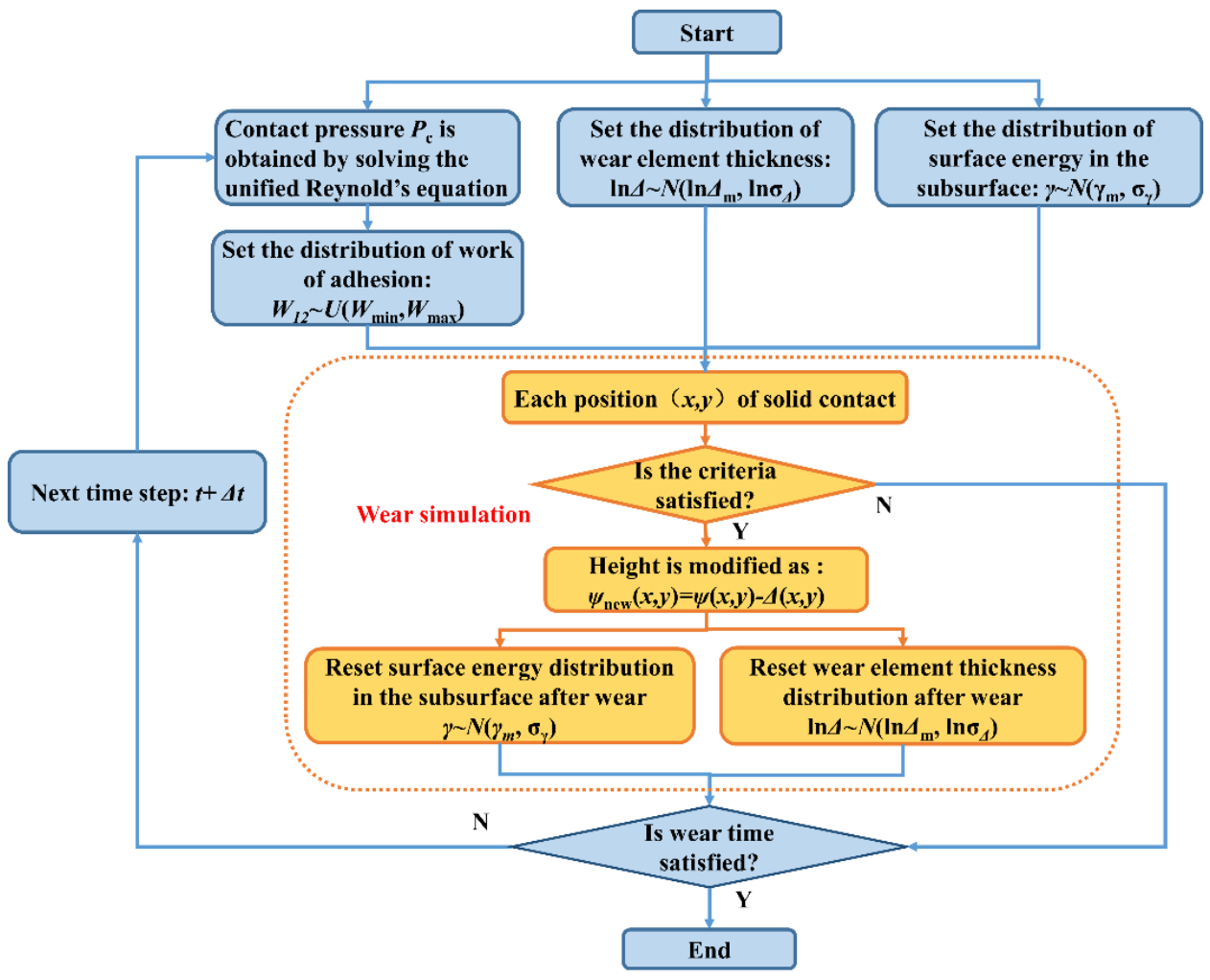

Fig. 2 Flow chart of wear simulation under mixed lubrication

Table 1 lists the input parameters for the simulation. The statistical parameters were selected from references $[25,27-28]$ for the steel materials. Tests with two reciprocating frequencies, $2 \mathrm{~Hz}$ and $0.2 \mathrm{~Hz}$, were performed. The lower speed test was done for experimental estimation of the magnitude of the conversion coefficient used in simulations, as described below.

\subsection{Estimation of conversion coefficient}

In this paper, an experimental method was used to approximately estimate the magnitude of conversion coefficient $\alpha$ in Eq. (1). Firstly, a low-speed friction test of the tribopair described in Section 3.1 was carried out to ensure the boundary lubrication state. The test conditions were the same as those in Section 3.1, except for the reciprocating frequency being set as $0.2 \mathrm{~Hz}$ instead of $2 \mathrm{~Hz}$. Table 2 shows the 3D topographies of the plate sample before and after the test, and the variation of friction coefficient recorded during the test. After the test, total wear volume $V$ in a period of friction time $T_{\mathrm{f}}$ of $5000 \mathrm{~s}$ was measured with a $3 \mathrm{D}$ profilometer as $8.18 \times 10^{5} \mu \mathrm{m}^{3}$, and frictional work $W_{\mathrm{f}}$ was calculated according to the friction coefficient measured during the test as $11.654 \mathrm{~J}$. Provided the statistical parameters for surface energy, work of adhesion and thickness of wear element are given as listed in Table 1, taking the wear element size as $1.17 \mu \mathrm{m}$, we can calculate the values of $A_{\mathrm{c}}$ and $A_{\mathrm{w}}$ as $1.1 \mu \mathrm{m}^{2}$ and $1.076 \mu \mathrm{m}^{2}$ 
respectively, and volume, $V_{\mathrm{w}}$, of a wear element with the mean thickness of $90 \mathrm{~nm}$ is $0.0487 \mu \mathrm{m}^{3}$. The number of equivalent wear elements $N_{\mathrm{w}}$ can be obtained as

$$
N_{\mathrm{w}}=\frac{V}{V_{\mathrm{w}}}
$$

When $\theta=1$, the adhesive wear criterion (1) can be re-written as

$$
\alpha=\frac{N_{\mathrm{w}}\left[\gamma_{\mathrm{m}} A_{\mathrm{w}}-0.5 A_{\mathrm{c}} W_{12 \mathrm{~m}}\right]}{W_{\mathrm{f}}}=\frac{W_{\mathrm{et}}}{W_{\mathrm{f}}}
$$

Substituting the values of $\gamma_{\mathrm{m}}$ and $W_{12 \mathrm{~m}}$ listed in Table 1 and the estimated values of $A_{\mathrm{c}}$, $A_{\mathrm{w}}, N_{\mathrm{w}}$ and $W_{\mathrm{f}}$ shown above, we can get an estimation of conversion coefficient $\alpha$ as $1.66 \times 10^{-6}$ for the steel ball-plate tribopair.

Table 1 Parameters for wear simulation

\begin{tabular}{lccc}
\hline Parameters & Symbol & Value & Unit \\
\hline Normal load & $W_{L}$ & 80 & $\mathrm{~N}$ \\
Lubricant viscosity at $40^{\circ} \mathrm{C}$ & $\eta_{0}$ & 0.048 & $\mathrm{~Pa} \cdot \mathrm{s}$ \\
Elastic modulus of disc & $E_{1}$ & 210 & $\mathrm{GPa}$ \\
Poisson's ratio of disc & $v_{1}$ & 0.3 & \\
Mean surface energy of disc & $\gamma_{\mathrm{m}}$ & 1.1 & $\mathrm{~J} / \mathrm{m}^{2}$ \\
Standard deviation of surface energy & $\sigma_{\gamma}$ & 0.055 & $\mathrm{~J} / \mathrm{m}^{2}$ \\
Root mean square roughness & $R_{\mathrm{q} 1}$ & 0.21 & $\mu \mathrm{m}$ \\
Elastic modulus of ball & $E_{2}$ & 210 & $\mathrm{GPa}$ \\
Poisson's ratio of ball & $v_{2}$ & 0.3 & \\
Stroke & $l_{s}$ & 5 & $\mathrm{~mm}$ \\
Frequency & $f_{r}$ & $2,0.2$ & $\mathrm{~Hz}$ \\
Average speed & $u_{\mathrm{e}}$ & 10 & $\mathrm{~mm} / \mathrm{s}$ \\
Maximum of work of adhesion & $W_{12 \mathrm{max}}$ & 0.22 & $\mathrm{~J} / \mathrm{m}^{2}$ \\
Minimum of work of adhesion & $W_{12 \mathrm{~min}}$ & 0 & $\mathrm{~J} / \mathrm{m}^{2}$ \\
Mean thickness of wear element & $\Delta_{m}$ & 90 & $\mathrm{~nm}$ \\
Standard deviation of thickness of wear element & $\sigma_{\Delta}$ & 43 & $\mathrm{~nm}$ \\
Friction coefficient of asperity contact & $\mu_{\mathrm{c}}$ & 0.12 & \\
\hline
\end{tabular}

Table 2 Results of friction experiments

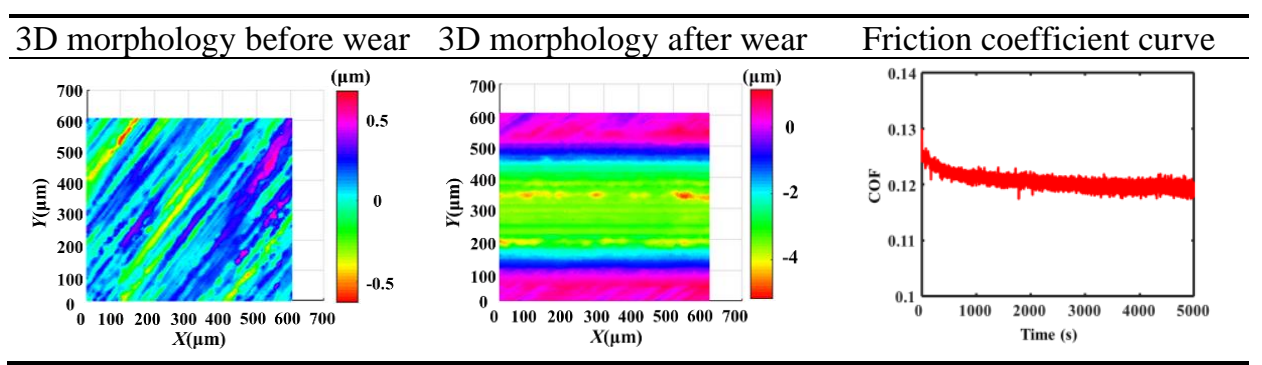




\subsection{Morphology evolution under mixed lubrication}

Due to the reciprocating friction, each contact point within the wear track on the steel plate slides twice in a motion cycle, and the relative sliding distance is one Hertz contact width. The wear area on the steel plate is only related to the number of friction cycles, being irrelevant to the actual reciprocating strokes. Therefore, in the wear simulation, the friction time is expressed as the number of cycles, and the sliding stroke is selected to be $800 \mu \mathrm{m}$, matching with the optical field range in the white light interferometery measurement. Figs. 3 and 4 show the thickness of primitive wear elements and surface energy distributions over the calculation domain, respectively.

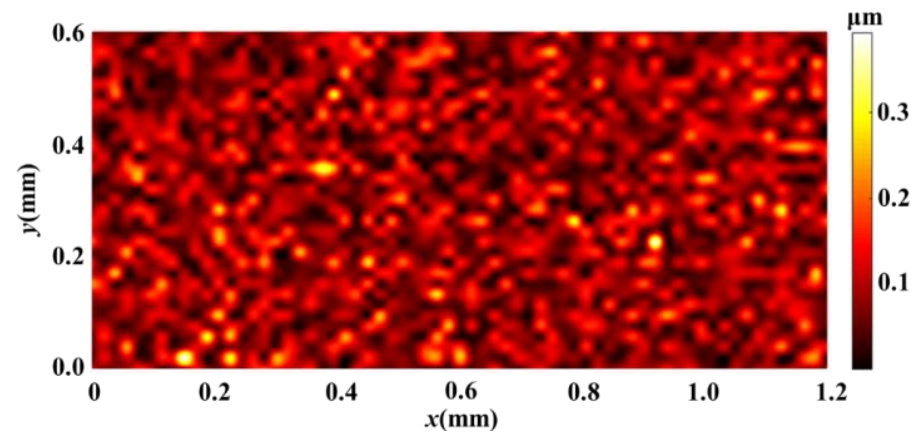

Fig. 3 Thickness distribution of basic wear element

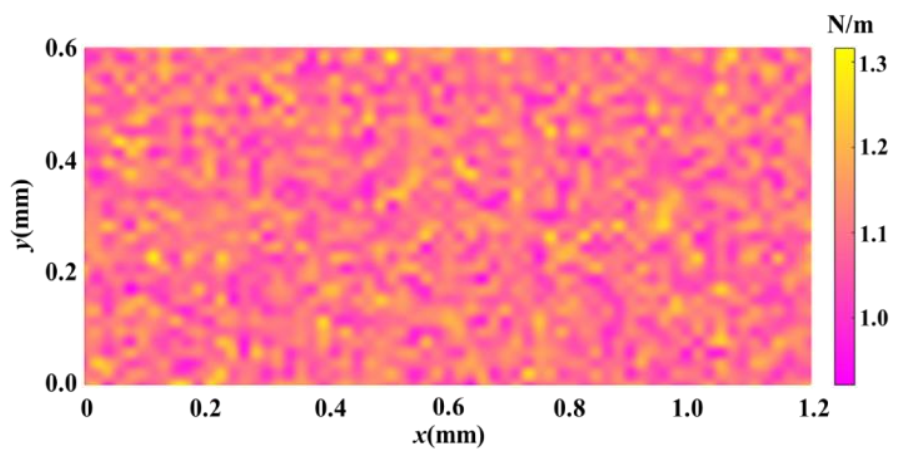

Fig. 4 Surface energy distribution on the surface

Fig. 5 shows the comparison of the evolution of 3D surface morphologies at different wear stages. It can be seen that with the increase of friction cycles, the portions with higher peaks decreases, and the wear marks become more and more clear. The original surface texture gradually fades, and new surface texture feature appears. 


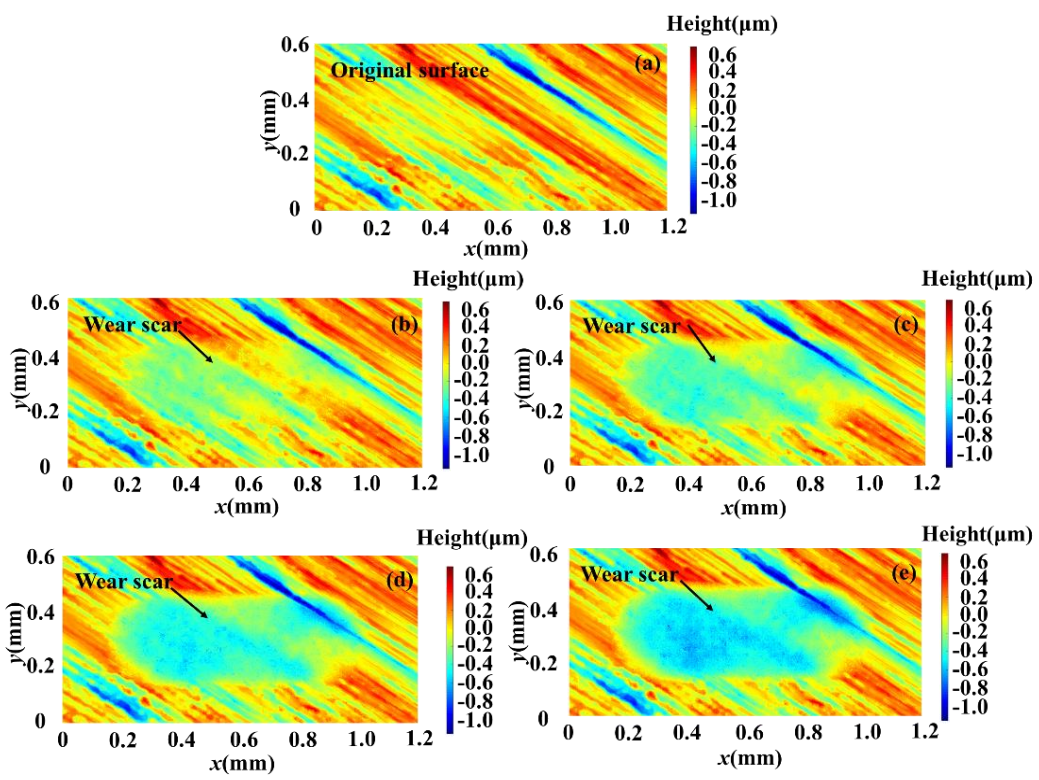

Fig. 5 Evolution of 3D morphology during friction process: (a) original surface (b) after 200 cycles (c) after 400 cycles (d) after 600 cycles (e) after 800 cycles

Fig. 6 shows that the profiles along the sliding direction in the middle section evolve with the sliding cycle. Just like in Fig. 5, it can be found that the rough peaks are gradually worn off during the wear process and the surface fluctuation presents evident randomness. In addition, it can be observed that at a fixed position, the height changes with great randomness, for the reason that the thickness of wear element was assumed to be stochastic in the modeling, which is in accord with observations in common wear surfaces.

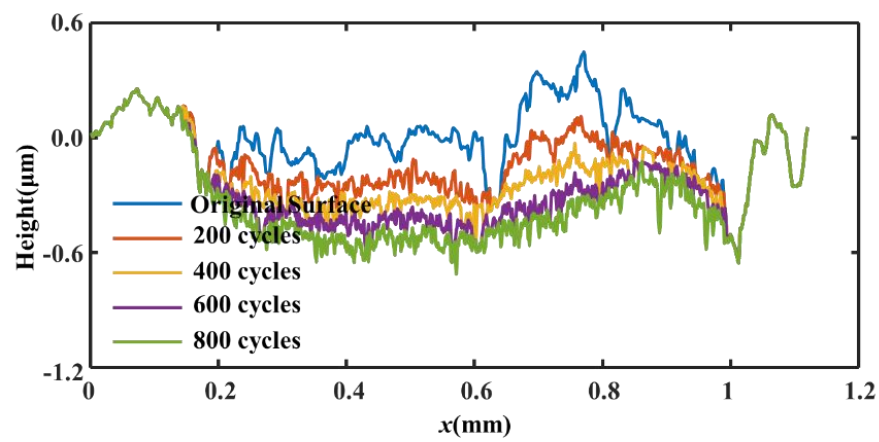

Fig. 6 2D profile evolution during friction process

Fig. 7 shows the change of cumulative wear volume during the wear process. It can be seen that with the increase of friction cycles, the slope of cumulative wear volume decreases gradually, indicating that the wear rate decreases gradually. The reason is that the solid bearing ratio keeps decreasing, which leads to a decrease in wear rate. In addition, 
with the decrease of $R_{\mathrm{q}}$, the difference between peak and valley of surface height decreases, resulting in a gradual decrease in pressure peak and frictional work at asperity junctions. Therefore, the shedding time of wear element increases and the wear rate decreases.

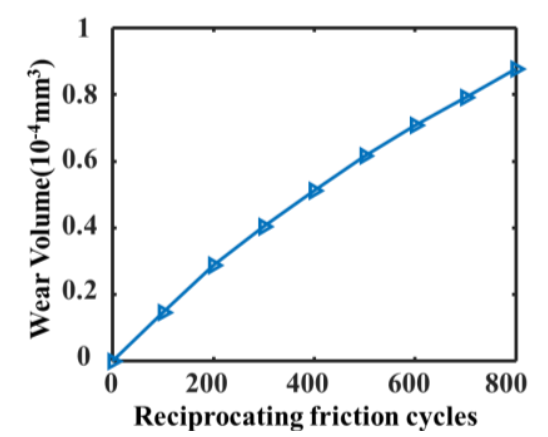

Fig. 7 Accumulative wear volume with friction cycles

Fig. 8 shows the change of $R_{\mathrm{q}}$ of the surface in the wear track during the wear process. It can be seen that with the increase of friction time, $R_{\mathrm{q}}$ decreases from $0.21 \mu \mathrm{m}$ to $0.13 \mu \mathrm{m}$, and the rate of decline gradually decreases, approximately reaching a stable state. This is because the peaks are apt to be worn away during the wear process. It should be noted that although the surface roughness approaches a stable value of $0.13 \mu \mathrm{m}$, it is still higher than the mean thickness of wear elements presumed in the simulation. This indicates that the set value of mean thickness of wear elements does not affect the roughness of worn surface remarkably. This is displayed more clearly in Fig. 9, where three different values, 45nm, $90 \mathrm{~nm}$ and $150 \mathrm{~nm}$, of the mean thickness of wear elements are set in wear simulations. It can be seen from Fig. 9(a) that when $\Delta_{\mathrm{m}}$ is $45 \mathrm{~nm}$, the surface morphology after 200 cycles does not deviate from the initial one too much. After 800 cycles, however, a new random surface profile forms, which is quite different from that of the virgin surface.

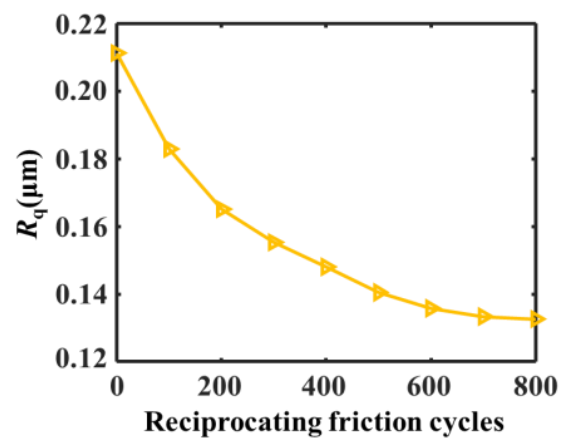

Fig. 8 Evolution of $R_{\mathrm{q}}$ in wear track with frictional cycle 

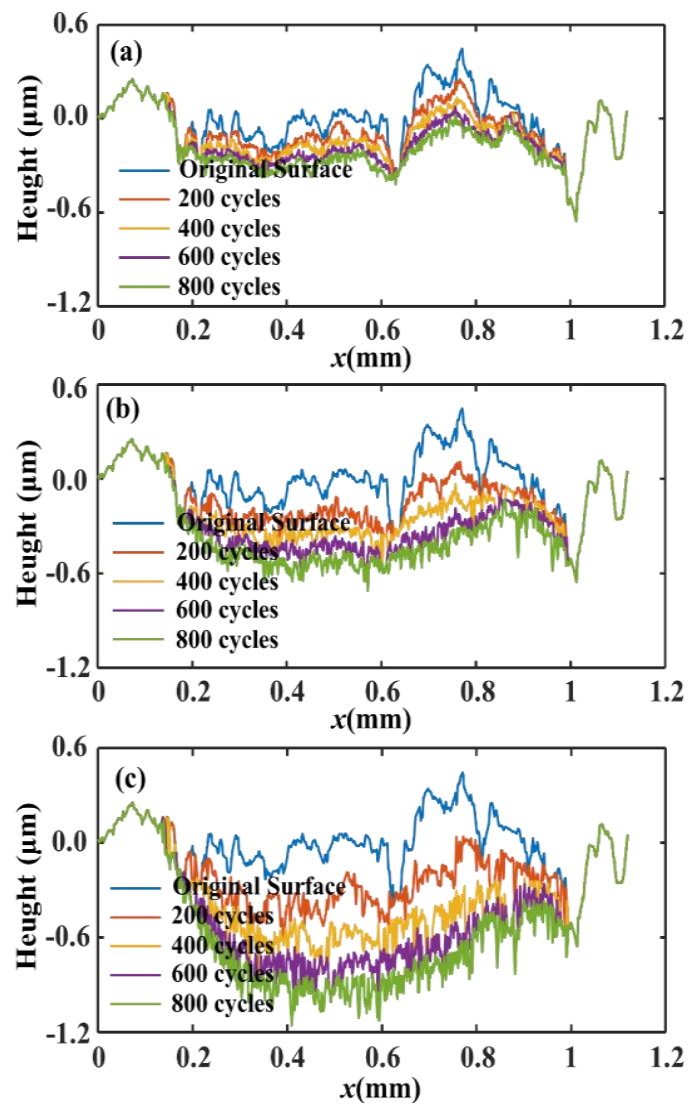

Fig. 9 Comparison of 2D profile evolution for different $\Delta_{\mathrm{m}}$ : (a) $\Delta_{\mathrm{m}}=45 \mathrm{~nm}$; (b) $\Delta_{\mathrm{m}}=90 \mathrm{~nm}$; (c) $\Delta_{\mathrm{m}}=150 \mathrm{~nm}$

From Figs. 9(b) and 9(c), it can be found that with the increase in the value of $\Delta_{\mathrm{m}}$, the morphology of worn surface rapidly changes apart from the initial topography. The original flat surface becomes more and more concave with the wear time, and the larger the $\Delta_{\mathrm{m}}$, the deeper the wear track for the same period of friction time. Fig. 10 shows accumulative wear volume for the different values of $\Delta_{\mathrm{m}}$ under the same test condition. It can be seen that wear rate decrease with friction time and wear volume slightly deviates from the linear relationship between the wear volume and the number of cycles. This can be explained by the evolution of surface roughness and severeness of asperity contact under mixed lubrication during the wear process. From Fig. 8, we can see that the root mean square roughness becomes smaller with friction cycles, which leads to an increasing film thickness ratio, or, in other words, to a better lubrication condition. In addition, from Figs. 6 and 9, we could see that surface height decreases and gradually forms a concave shape of the plate surface with the friction cycles. This means that the initial ball-on-flat nonconformal concentrated contact gradually changes to a more or less conformal contact with a wider and wider contact area, leading to wider but lower contact pressure. From Eq. (1), we can find that if pc decreases, it cost a longer $t$ to satisfy the condition of $\theta>1$. 

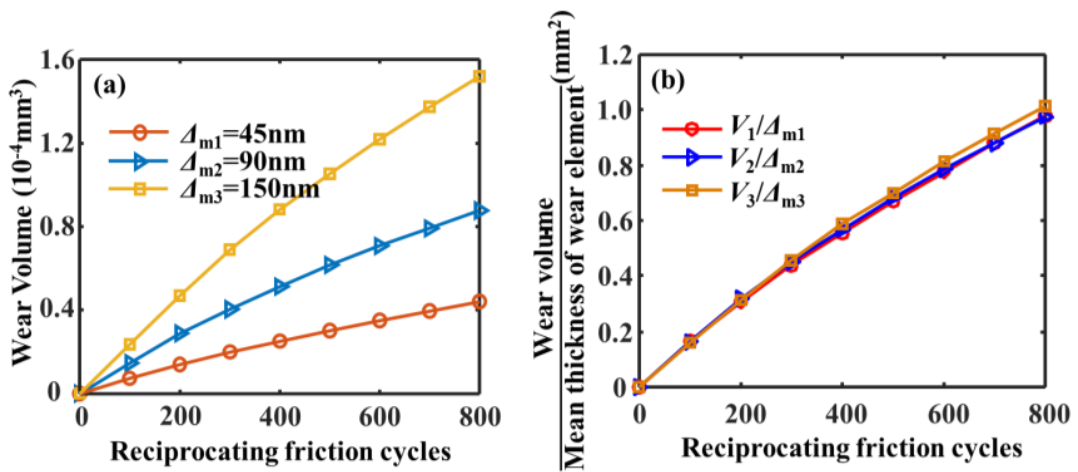

Fig. 10 Wear volume vs. frictional cycle for different mean thickness of wear element

\subsection{Comparison of wear process between experiments and simulations}

A reciprocating friction and wear tester (Rtec Instrument, USA) was used to conduct in-situ measurement of evolution of three-dimensional surface morphology during wear under mixed lubrication condition. The test diagram is shown in Fig. 11. The upper sample is a bearing steel ball with diameter of $12.7 \mathrm{~mm}$, the RMS surface roughness is $0.014 \mu \mathrm{m}$, and the vickers hardness is $810 \mathrm{HV}$. The lower sample is a carbon steel plate with RMS surface roughness of $0.16 \mu \mathrm{m}$ and vickers hardness of $395 \mathrm{HV}$.

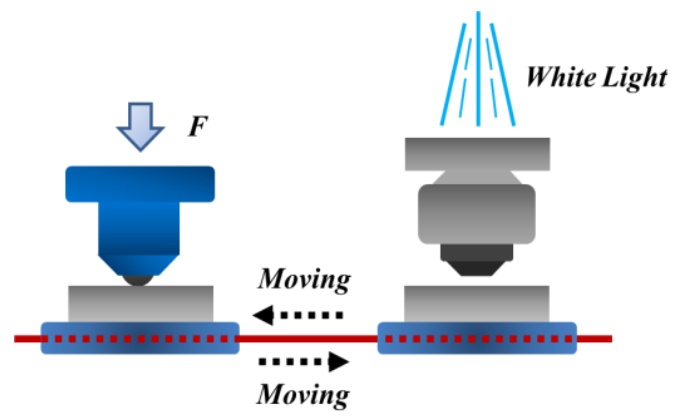

Fig. 11 Diagram of friction and wear test

The experimental procedure was as follows. Firstly, the sample table with the lower sample installed was moved to the position under the white light interferometer module, and the initial 3D surface morphology of the sample was recorded. Then the sample table was moved along the linear guide rail to the friction module position, and a certain amount of PAO lubricating oil was dropped at the sample surface to be tested. After the upper sample touched with the lower sample, it was loaded to the set load of $80 \mathrm{~N}$, and then the reciprocating sliding was started. The sliding friction was suspended every 100 reciprocating cycles. The upper sample was lifted and it gets rid of contact with the lower sample surface. The residual oil on the surface of the lower sample was scrubbed with acetone and dried with compressed nitrogen gas. Then, the cleaned sample was shifted to the position under the white light interferometer. After the morphology measurement, the sample table was translated back to the friction test position along the guide rail, 
re-lubricated the sample, applied the normal load, and re-started the friction test at the same reciprocating motion speed. The stroke of the motion was $5 \mathrm{~mm}$, and the frequency set at 2 Hz. 10 times magnification objective lens was used for wear morphology measurement.

The advantage of the start-stop friction and wear test protocol is to avoid the detachment of the samples for measurement of wear morphology; hence no need was there to re-install the specimen, which may cause misalignment problems between two successive test phases.

Fig. 12 shows the comparisons of cross-sectional profiles between the experimental and the simulation results for the friction cycles of 300,600 and 900. It can be seen that the consistence between the simulated wear profile and the experimental measurements is reasonable, and major features of the predicted and measured topographies are in accord with each other.

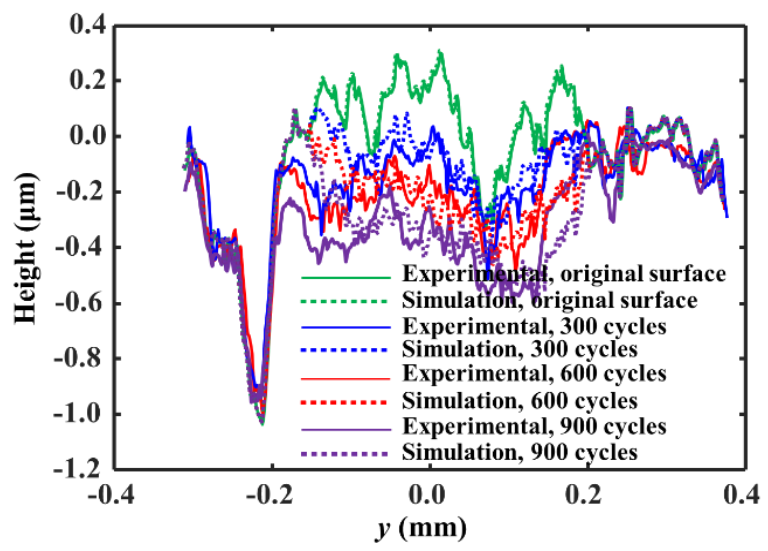

Fig. 12 Comparison of morphology evolution between experiments and simulations

However, there are some discrepancies between the experimental and simulation results, as shown in Fig. 12. Both the mean lines and fluctuation magnitudes of the predicted profiles do not fit in very well with the experimental ones for a given friction cycle. There are several possible reasons for the discrepancies. Among the reasons are inaccurate inputs of the parameters of surface energy, work of adhesion, thickness of wear element and the conversion coefficient used in the simulations. Secondly, in the wear model, it is assumed that only adhesive wear happens and the wear particles would escape from the contact area quickly as soon as they formed. However, in a real wear process, some of wear particles may trap at the contact area, resulting in abrasive wear. Third, some of adhesive wear elements would attach on the counterpart surface till loosing from the surface later, these transferred materials would change the morphology and work of adhesion, which are not considered in the wear simulation. Further discussions on the limitations of the proposed wear criterion are given below.

\section{DISCUSSIONS}

Differing from the Archard law or other previous wear equations which relate total loss of material in terms of volume, mass or depth with material property and working condition explicitly, the wear criterion proposed in the study provides only a necessary condition for a wear element to generate. The loss of material at certain friction period is implicitly expressed by summation of the volume of all wear elements which satisfy the 
necessary condition during that period, provided that the probability density function of the thickness of wear elements is known. Another characteristic of the wear criterion is that it accounts the material degradation owing to frictional work. Stochastic feature of local wear observed in practice is also accounted by introducing random distributions of the parameters of surface energy, work of adhesion and the wear element thickness.

The wear criterion in Eq. (1) can apply to analyzing the wear of both bodies, A and B, in contact. In that case, the surface energy in the denominator in wear criterion (1) takes as the value of the surface energy A or B respectively, while the numerator and the term of frictional work in the denominator for body B are the same as those for body A.

Both Rabinowicz's wear particle size equation and our local wear criterion include the term of work of adhesion and thus emphasize its vital role in adhesive wear. In this sense, they are similar. But there are several distinctions between them. First, as described in introduction of the manuscript, the Rabinowicz equation was derived from the assumption that a loose wear particle forms when the release of elastic deformation energy stored in a particle overcomes the cost of the increase in energy due to the detachment of that particle from the attached surface, which equals to the work of adhesion. In the derivation of our criterion, however, we based on the material degradation mechanism as expressed in the bracket in the denominator of Eq. (1), which comes from fatigue and fracture mechanics, rather than the elastic energy restoration mechanism as Rabinowicz used. In consequence, it needs a damage accumulation or material surface energy degradation stage $[0, t]$, as expressed in the integration of frictional work over the period $[0, t]$ in the denominator, for a wear element to form, while such a degradation stage is not needed by Rabinowicz equation. Secondly, Rabinowicz assumed that the shape of particles is hemisphere for the sake of simplicity in mathematical derivation. In our model, we did not make any assumptions on the size and shape of wear particles. Instead, we assumed the shape of wear elements is spherical crown for the sake of simplicity to estimate $A_{\mathrm{c}}$ and $A_{\mathrm{w}}$ in Equation (1). The size and shape of wear particles are determined by the connectivity of wear elements. Thirdly, we introduced probability distribution functions to the material parameters of work of adhesion $W_{12}$ and surface energy $\gamma$, while Rabinowicz equation is deterministic.

However, it should be mentioned that the wear criterion in Eq. (1) does not tell us any information of the thickness of wear elements, which was necessary and assumed in the simulations presented above. A detailed microscopic analysis of damage initiation and propagation is needed to reveal where fracture of material most probably occurs and their statistic distribution for a macro-tribosystem.

\section{CONCLUSIONS}

Based on the understanding of adhesive wear mechanism, a novel fracture-induced adhesive wear criterion has been proposed. The effects of work of adhesion and surface energy degradation on adhesive wear are taken into account in the criterion. Moreover, the stochastic distributions of these physical properties of materials are also considered. Coupled with the deterministic mixed lubrication theory, the wear criterion has been applied to predicting the wear process of a mixed lubricated point contact in sliding motion. It has been shown that morphology evolution of surfaces and wear particles formation during wear process can be simulated by using the proposed wear criterion and simulation procedure. Comparison of simulation results with experiment measurements has been done, and the agreement between them is reasonable. 
Acknowledgements: This work was supported by the National Natural Science Foundation of China (NSFC) under grant No. 51635009 and the Ministry of Industry and Information Technology, China, with grant number JSZL2017213B002.

\section{REFERENCES}

1. Harris, T.A., Kotzalas, M.N., 2007, Rolling Bearing Analysis (5th Edition): Advanced Concepts of Bearing Technology, Taylor \& Francis.

2. Erdemir, A., Martin, J.M., Luo, J., 2020, Pradeep, N., Superlubricity (2nd Edition), Elsevier.

3. Zhang, Y., Kovalev, A., Meng, Y., 2018, Combined effect of boundary layer formation and surface smoothing on friction and wear rate of lubricated point contacts during normal running-in processes, Friction, 6, pp. 274-288.

4. Zhao, D., Lu, X., Molinari, J.F., 2013, Chemical mechanical polishing: theory and experiment, Friction, 1, pp. 306-326.

5. Holm, R, 1946, Electric Contacts: Theory and Applications, Stockholm.

6. Meng, H.C., Ludema, K.C., 1995, Wear models and predictive equations: their form and content, Wear, 181-183, pp. 443-457.

7. Rhee, S.K., 1970, Wear equation for polymers sliding against metal surfaces, Wear, 16, pp. 431-445.

8. Archard, J.F., 1953, Contact and rubbing of flat surfaces, J. Applied Phys., 24, pp. 981-988.

9. Rabinowicz, E., 1961, Influence of surface energy on friction and wear phenomena, J. Applied Phys., 32, pp. 1440-1444.

10. Rabinowicz, E., 1964, Practical uses of the surface energy criterion, Wear, 7, pp. 9-22.

11. Suh, N.P., 1973, The delamination theory of wear, Wear, 25, pp. 111-124.

12. Quinn, T., 1971, Oxidational wear, Wear, 18, pp. 413-419.

13. Carpick R. W., Salmeron M., 1997, Scratching the surface: Fundamental investigations of tribology with atomic force microscopy, Chem Rev, 97, pp.1163-1194.

14. Greer, J.R., Nix, W.D., 2005, Size dependence of mechanical properties of gold at the sub-micron scale, Applied Physics A, 80, pp. 1625-1629.

15. Gotsmann, B., Lantz, M.A., 2008, Atomistic wear in a single asperity sliding contact, Phys Rev Lett, 101, pp. 125501 .

16. Hase, A., Mishina, H., 2009, Wear elements generated in the elementary process of wear, Tribology International, 42, pp. 1684-1690.

17. Vargonen, M., Yang, Y.J., Huang, L.P., Shi, Y.F., 2013, Molecular simulation of tip wear in a single asperity sliding contact, Wear, 307, pp. 150-154.

18. Aghababaei, R., Warner, D.H., Molinari, J.F., 2016, Critical length scale controls adhesive wear mechanisms, Nature. Communications, 7, 11816.

19. Yu, D., Wang, J., Ma, M., Meng, Y., 2020, Effect of surface energy on shearing of metal asperities contact at nanoscale, The Journal of Physical Chemistry C, 124, pp. 27436-27441.

20. Zhou, C., Beyerlein, I.J., LeSar, R., 2011, Plastic deformation mechanisms of fcc metals as small scales, Acta Materiallia, 59, pp. 7673-7682.

21. El-Awady, J., 2014, Unravelling the physics of size-dependent dislocation-mediated plasticity, Nature Communications, 6, pp. 5926.

22. Popov, V., Pohrt, R., 2018, Adhesive wear and particle emission: numerical approach based on asperity-free formulation of Rabinowicz criterion, Friction, 6, pp. 260-273.

23. Tan, Y., Zhang, L., Hu, Y., 2014, A wear model of plane sliding pairs based on fatigue contact analysis of asperities, Tribology Transactions, 58, pp. 148-157.

24. Isiet, M., Miskovic, I., Miskovic, S., 2021, Review of peridynamic modelling of material failure and damage due to impact, Intern. J. of Impact Engineering, 147, 103740.

25. Rabinowicz, E., 1995, Friction and wear of materials, Second Edition, John Wiley \& Sons, Inc.

26. Hu, Y., Zhu, D., 2000, A full numerical solution to the mixed lubrication in point contacts, Journal of Tribology, 122, pp. 481-491.

27. Suh, N.P., 1977, An overview of the delamination theory of wear, Wear, 44, pp. 39-56.

28. Popov, V., Gerve, A., Kehrwald, B., et al., 2000, Simulation of wear in combustion engines, Computational Materials Science, 19, pp. 285-291. 